\title{
Balancedness conditions for exact games
}

\author{
Péter Csóka • P. Jean-Jacques Herings • \\ László Á. Kóczy
}

Received: 10 June 2010 / Accepted: 24 February 2011 / Published online: 20 March 2011

(C) The Author(s) 2011. This article is published with open access at Springerlink.com

\begin{abstract}
We provide two new characterizations of exact games. First, a game is exact if and only if it is exactly balanced; and second, a game is exact if and only if it is totally balanced and overbalanced. The condition of exact balancedness is identical to the one of balancedness, except that one of the balancing weights may be negative, while for overbalancedness one of the balancing weights is required to be non-positive and no weight is put on the grand coalition. Exact balancedness and overbalancedness are both easy to formulate conditions with a natural game-theoretic interpretation and are shown to be useful in applications. Using exact balancedness we show that exact games are convex for the grand coalition and we provide an alternative proof that the classes of convex and totally exact games coincide. We provide an example of a game that is totally balanced and convex for the grand coalition, but not exact. Finally we relate classes of balanced, totally balanced, convex for the grand coalition, exact, totally exact, and convex games to one another.
\end{abstract}

Keywords Totally balanced games · Exact games · Convex games

\footnotetext{
P. Csóka

Department of Finance, Corvinus University of Budapest, Budapest, Hungary

e-mail: peter.csoka@uni-corvinus.hu

P. J.-J. Herings $(\varangle)$

Department of Economics, Maastricht University, P.O. Box 616, 6200 MD, Maastricht, The Netherlands

e-mail: P.Herings@maastrichtuniversity.nl
}

L. Á. Kóczy

Institute of Economics of the Hungarian Academy of Sciences and Óbuda University,

Budapest, Hungary

e-mail: Koczy@econ.core.hu 


\section{JEL Classification $\quad \mathrm{C} 71 \cdot \mathrm{C} 61$}

\section{Introduction}

It is well-known that the core in a transferable utility game is non-empty if and only if the game is balanced (Bondareva 1963; Shapley 1967). ${ }^{1}$ The core of every subgame of a transferable utility game is non-empty if and only if the game is totally balanced. Totally balanced games turn up in a wide range of applications. They coincide with market games (Shapley and Shubik 1969); also with a special case of market games with a continuum of indivisible commodities (Legut 1990); they are equivalent to a class of maximum flow problems (Kalai and Zemel 1982a); and also to permutation games of less than four players (Tijs et al. 1984). Moreover, totally balanced games are generated by linear production games (Owen 1975), generalized network problems (Kalai and Zemel 1982b), and controlled mathematical programming problems (Dubey and Shapley 1984).

In this paper we study a subclass of totally balanced games, exact games (Schmeidler 1972). Exact games have the following applications. Calleja et al. (2005) show that the class of multi-issue allocation games equals the class of non-negative exact games. Csóka et al. (2009) demonstrate that the class of exact games coincides with the class of risk allocation games with no aggregate uncertainty. Branzei et al. (2009) show that convex multi-choice games are exact. It has been shown that largeness, extendability and exactness coincide in chain-component additive games (van Velzen et al. 2008) and also in simple flow games (Fang et al. 2010). Casas-Méndez et al. (2003) prove that if one takes any exact game and a coalitional structure, then the resulting coalitional game will be quasi balanced. If a game is quasi balanced, then their proposed solution concept, the coalitonal $\tau$-value can be defined.

We provide a set of linear programming problems by which one can easily check whether a game is exact or not. Using the dual we develop two new characterizations of exact games complementing those by Schmeidler (1972) and Azrieli and Lehrer (2005).

The first characterization of the class of exact games is the condition of exact balancedness. One interpretation of balancedness is the following. Each coalition forms for a non-negative amount of time being such that each player works exactly one unit of time together in the coalitions to which he belongs. In doing so the players cannot generate more value than when all work a full unit of time for the grand coalition. The condition for exact balancedness is identical to the one of balancedness, except that one coalition is allowed to form during a negative amount of time. We can think of this as the other coalitions working overtime and paying the opportunity cost of doing so to the coalition that forms during a negative amount of time. We show that exact balancedness implies total balancedness.

The second characterization spells out what more than total balancedness is needed to obtain exactness. It says that a game is exact if an only if it is totally balanced and

\footnotetext{
1 Carpente et al. (2010) show that the truncated core in a transferable utility game is non-empty if and only if the game is $t$-balanced.
} 
overbalanced. In case of overbalancedness, no weight is put on the grand coalition and one coalition works a non-negative amount of overtime. From this characterization it follows immediately that an exact game is totally balanced.

We demonstrate the simplicity of exact balancedness in applications. Biswas et al. (1999) show that totally exact games are convex (and that convex games are totally exact). Using exact balancedness we provide an alternative proof of this result.

We also study games that are convex for the grand coalition, where convexity is only required for coalitions whose union is the set of all players. We show that exact balancedness implies that a game is convex for the grand coalition, which leads to the result that exact games in case of at most three players are convex. Using the intuition behind exact balancedness, we provide an example of a game which is both totally balanced and convex for the grand coalition, but which is not exact.

The structure of the paper is as follows. We start with the notation and the necessary definitions. In Sect. 3 we study the balancedness conditions for exact games. In Sect. 4 we demonstrate the usefulness of our characterizations of exact games. We conclude the paper with a summary of how the classes of balanced, totally balanced, convex for the grand coalition, exact, totally exact, and convex games are related to one another.

\section{Notation and definitions}

Let $N=\{1, \ldots, n\}$ denote a finite set of players, $\mathcal{N}$ is the collection of non-empty subsets of $N$, and $\mathcal{D}$ is the collection of non-empty subsets of a coalition $D \in \mathcal{N}$. $A$ value function $v: 2^{N} \rightarrow \mathbb{R}$ satisfying that $v(\emptyset)=0$ gives rise to a cooperative game with transferable utility (game, for short) $(N, v)$. Let $\Gamma$ denote the set of games with $n$ players. An allocation is a vector $x \in \mathbb{R}^{n}$, where $x_{i}$ is the payoff of player $i \in N$. For a coalition $C \in \mathcal{N}$, let $x(C)=\sum_{i \in C} x_{i}$. An allocation $x \in \mathbb{R}^{n}$ is called efficient if $x(N)=v(N)$, individually rational if $x_{i} \geq v(\{i\})$ for all $i \in N$, and coalitionally rational if $x(C) \geq v(C)$ for all $C \in \mathcal{N}$. The core is the set of efficient and coalitionally rational allocations.

For each $C \in \mathcal{N}, a(C) \in \mathbb{R}^{n}$ is the membership vector in $C$, where $a_{i}(C)=1$ if $i \in C$ and $a_{i}(C)=0$ otherwise. We define $\mathbb{R}_{+}=[0, \infty)$.

Definition 2.1 A balanced vector of weights is $\left(\lambda_{C}\right)_{C \in \mathcal{N}}$ such that $\lambda_{C} \in \mathbb{R}_{+}$for all $C \in \mathcal{N}$ and $\sum_{C \in \mathcal{N}} \lambda_{C} a(C)=a(N)$. A game $(N, v)$ is balanced if $\sum_{C \in \mathcal{N}} \lambda_{C} v(C) \leq$ $v(N)$ for all balanced vectors of weights $\left(\lambda_{C}\right)_{C \in \mathcal{N}}$.

Let $\Gamma_{\mathrm{b}}$ denote the class of balanced games with $n$ players. A well-known interpretation of balancedness is that if the players distribute one unit of working time to any coalition and each coalition is active during $\lambda_{C}$ time units, then the players cannot generate more value than $v(N)$, when working a full unit of time for the grand coalition. Balancedness is a necessary and sufficient condition for the core in a transferable utility game to be non-empty (Bondareva 1963; Shapley 1967); Predtetchinski and Herings (2004) provide the corresponding necessary and sufficient balancedness conditions for non-transferable utility games.

For a game $(N, v)$ and a coalition $D \in \mathcal{N}$ the subgame $\left(D, v^{D}\right)$ is obtained by restricting $v$ to subsets of $D$. 
Definition 2.2 A game $(N, v)$ is totally balanced if for every $D \in \mathcal{N}$ its subgame $\left(D, v^{D}\right)$ is balanced, that is, if for all $D \in \mathcal{N}$ and for all vectors $\left(\lambda_{C}\right)_{C \in \mathcal{D}}$ such that $\lambda_{C} \in \mathbb{R}_{+}$for all $C \in \mathcal{D}$ and $\sum_{C \in \mathcal{D}} \lambda_{C} a(C)=a(D)$, we have $\sum_{C \in \mathcal{D}} \lambda_{C} v(C) \leq$ $v(D)$.

In a totally balanced game every subgame has a non-empty core. Let $\Gamma_{\mathrm{tb}}$ denote the class of totally balanced games with $n$ players.

Schmeidler (1972) introduces exact games.

Definition 2.3 A game $(N, v)$ is exact if for each $C \in \mathcal{N}$ there exists a core allocation $x$ such that $x(C)=v(C)$.

Let $\Gamma_{\mathrm{e}}$ denote the class of exact games with $n$ players. Convex games (Shapley 1971) can be defined as follows.

Definition 2.4 A game $(N, v)$ is convex if for all $S, T \in 2^{N}$ we have $v(S)+v(T) \leq$ $v(S \cup T)+v(S \cap T)$.

Let $\Gamma_{\mathrm{c}}$ denote the class of convex games with $n$ players. Convex games are exact (Schmeidler 1972). We now introduce the new notion of convexity for the grand coalition.

Definition 2.5 A game $(N, v)$ is convex for the grand coalition if for all $S, T \in 2^{N}$ such that $S \cup T=N$ we have $v(S)+v(T) \leq v(N)+v(S \cap T)$.

Let $\Gamma_{\text {cg }}$ denote the set of games which are convex for the grand coalition. Following Biswas et al. (1999), we define totally exact games analogously to totally balanced games.

Definition 2.6 A game $(N, v)$ is totally exact if for every $D \in \mathcal{N}$ its subgame $\left(D, v^{D}\right)$ is exact.

Let $\Gamma_{\text {te }}$ denote the class of totally exact games with $n$ players.

\section{Exact games and balancedness}

Consider a game $(N, v) \in \Gamma$. For each coalition $D \in \mathcal{N}$ we develop a linear programming problem related to the game $(N, v)$. The linear program is such that whenever $(N, v)$ is exact, any optimal solution $x^{*}$ is a core allocation satisfying $x^{*}(D)=v(D)$. We denote the linear program by $\mathrm{P}_{v, D}$ and its dual by $\mathrm{P}_{v, D}^{*}$.

$$
\begin{aligned}
& \min a(N) x \quad \max \sum_{C \in \mathcal{N}} \lambda_{C} v(C) \\
& \text { s.t. } \\
& \text { s.t. } \\
& \left(\mathrm{P}_{v, D}\right) \quad a(C) x \geq v(C), \quad C \in \mathcal{N} \backslash\{D\} \quad\left(\mathrm{P}_{v, D}^{*}\right) \quad \sum_{C \in \mathcal{N}} \lambda_{C} a(C)=a(N) \\
& a(D) x=v(D) \quad \lambda_{C} \in \mathbb{R}_{+}, \quad C \in \mathcal{N} \backslash\{D\} \\
& x \in \mathbb{R}^{n} \\
& \lambda_{D} \in \mathbb{R} \text {. }
\end{aligned}
$$


Let us discuss the primal. The first part of the feasibility constraints requires that $x$ is coalitionally rational for all coalitions but coalition $D$. The second part of the feasibility constraints requires that $x(D)=v(D)$, that is, $x$ should be efficient in the subgame $\left(D, v^{D}\right)$, so a feasible solution yields a core element in the subgame $\left(D, v^{D}\right)$.

If $\left(D, v^{D}\right)$ is a balanced game, then the set of feasible solutions is non-empty, since we have a core element in the subgame $\left(D, v^{D}\right)$ and the other elements of a feasible solution can be chosen sufficiently large. In this case the set of optimal solutions of $\mathrm{P}_{v, D}$ is also non-empty since the set of feasible solutions is bounded from below. If $\left(D, v^{D}\right)$ is not balanced, then the set of feasible solutions of $\mathrm{P}_{v, D}$ is empty, since then $\left(D, v^{D}\right)$ has no core allocations. If $x^{*}$ is an optimal solution of the primal, then $o_{v, D}=a(N) x^{*}$ is the value of the optimal solution, which by the duality theorem of linear programming is the same as the optimal objective value of the dual. If $\mathrm{P}_{v, D}$ has no optimal solutions, then we define $o_{v, D}=\infty$. The linear programming problems are related to exactness as follows.

Proposition 3.1 A game $(N, v) \in \Gamma$ is exact if and only if for every $D \in \mathcal{N}$ we have that $o_{v, D}=v(N)$.

Proof $(\Rightarrow)$ The proof is by contradiction. Take any exact game $(N, v) \in \Gamma_{\mathrm{e}}$ and assume that there is a coalition $D \in \mathcal{N}$ such that $o_{v, D} \neq v(N)$. Let $x^{*}$ be an optimal solution of $\mathrm{P}_{v, D}$. Using the feasibility constraints of the primal we have that $a(N) x^{*}>$ $v(N)$. As $(N, v)$ is exact there exists a core element $x$ such that $x(D)=v(D)$ and $x(N)=v(N)$. The vector $x$ satisfies the feasibility constraints and has objective value $a(N) x=v(N)<a(N) x^{*}$, a contradiction.

$(\Leftarrow)$

Consider some $D \in \mathcal{N}$ and let $x^{*}$ be an optimal solution of $\left(\mathrm{P}_{v, D}\right)$, so $a(N) x^{*}=$ $v(N)$, that is $x^{*}$ is efficient. The efficiency and the feasibility constraints imply that $x^{*}$ is a core allocation of $(N, v)$ with $x(D)=v(D)$. Thus $(N, v)$ is exact.

Let us continue by analyzing the dual, $\mathrm{P}_{v, D}^{*}$. The value of its optimal solution is at least $v(N)$, since $v(N)$ is obtained by setting $\lambda_{N}=1$ and $\lambda_{C}=0$ for all $C \in \mathcal{N} \backslash\{N\}$. It follows from optimality that $o_{v, D}=v(N)$ if and only if for all feasible solutions of $\mathrm{P}_{v, D}^{*}$ the value of the objective function is not larger than $v(N)$. Using this observation, Proposition 3.1, and the duality theorems in linear programming we have the following corollary.

Corollary 3.2 A game $(N, v) \in \Gamma$ is exact if and only if for every $D \in \mathcal{N}$ and for each vector $\left(\lambda_{C}\right)_{C \in \mathcal{N}}$ such that for $C \in \mathcal{N} \backslash\{D\}, \lambda_{C} \in \mathbb{R}_{+}, \lambda_{D} \in \mathbb{R}$, and $\sum_{C \in \mathcal{N}} \lambda_{C} a(C)=a(N)$ we have $\sum_{C \in \mathcal{N}} \lambda_{C} v(C) \leq v(N)$.

In Theorem 3.5 below, we rewrite the conditions in Corollary 3.2 using exact balancedness, defined as follows.

Definition 3.3 An exactly balanced vector of weights is a vector $\left(\lambda_{C}\right)_{C \in \mathcal{N}}$ such that for some $D \in \mathcal{N}, \lambda_{D} \in \mathbb{R}$, for all $C \neq D, \lambda_{C} \in \mathbb{R}_{+}$, and $\sum_{C \in \mathcal{N}} \lambda_{C} a(C)=a(N)$. A game $(N, v)$ is exactly balanced if $\sum_{C \in \mathcal{N}} \lambda_{C} v(C) \leq v(N)$ for all exactly balanced vectors of weights. 
Let $\Gamma_{\text {eb }}$ denote the class of exactly balanced games with $n$ players. Notice that the only difference to the condition of balancedness is that one weight, $\lambda_{D}$ can be arbitrary (negative, zero, or positive). Due to this extra freedom, the set of exactly balanced vectors is larger than the set of balanced vectors, hence it is more difficult to satisfy exact balancedness than balancedness. To interpret a negative weight, consider the following example.

Example 3.4 Take an arbitrary game $(N, v)$ with player set $N=\{1,2,3,4\}$. If all other balancing weights are set to zero, then setting $\lambda_{\{1\}}=1, \lambda_{\{2\}}=1, \lambda_{\{1,3\}}=$ $1, \lambda_{\{2,4\}}=1, \lambda_{\{1,2\}}=-1$ constitutes an exactly balanced vector of weights. For exact balancedness

$$
v(\{1\})+v(\{2\})+v(\{1,3\})+v(\{2,4\})-v(\{1,2\}) \leq v(\{1,2,3,4\})
$$

should hold. Equation (1) can be interpreted as follows. Players in the coalition with the negative weight, here coalition $(\{1,2\})$, work overtime, each member allocating $-\lambda_{\{1,2\}}$ extra time among the remaining coalitions. Equation (1) says that when the opportunity cost of this extra effort is subtracted, the whole set of players cannot generate more value than the grand coalition. This is even more apparent after rearranging:

$$
v(\{1\})+v(\{2\})+v(\{1,3\})+v(\{2,4\}) \leq v(\{1,2,3,4\})+v(\{1,2\}),
$$

thus working for the grand coalition and working overtime in the coalition with the negative weight is preferable.

Exact balancedness is a necessary and sufficient condition for a game to be exact, as the following theorem claims.

Theorem 3.5 A game $(N, v) \in \Gamma$ is exact if and only if it is exactly balanced, that is $\Gamma_{\mathrm{e}}=\Gamma_{\mathrm{eb}}$.

Proof This follows immediately from Corollary 3.2.

Exact games can also be characterized by total balancedness and overbalancedness, to be defined next.

Definition 3.6 An overbalanced vector of weights is a vector $\left(\mu_{C}\right)_{C \in \mathcal{N} \backslash\{N\}}$ such that $\mu_{C} \in \mathbb{R}_{+}$for all $C \in \mathcal{N} \backslash\{N\}$ and $\sum_{C \in \mathcal{N} \backslash\{D, N\}} \mu_{C} a(C)=a(N)+\mu_{D} a(D)$ for some $D \in \mathcal{N}$. A game $(N, v)$ is overbalanced if $\sum_{C \in \mathcal{N} \backslash\{D, N\}} \mu_{C} v(C) \leq v(N)+\mu_{D} v(D)$ for all overbalanced vectors of weights.

Let $\Gamma_{\mathrm{ob}}$ denote the class of overbalanced games with $n$ players. The difference between balancedness and overbalancedness is that in the case of overbalancedness, no weight is put on the grand coalition and one weight is non-positive (after rearranging we get $-\mu_{D} \leq 0$ ). As in Eq. (2), the overbalancedness condition requires that working for the grand coalition and working a non-negative amount of overtime in coalition $D$ is the best choice players can make. 
Theorem 3.7 A game $(N, v) \in \Gamma$ is exact if and only if it is totally balanced and overbalanced, that is $\Gamma_{\mathrm{e}}=\Gamma_{\mathrm{tb}} \cap \Gamma_{\mathrm{ob}}$.

Proof We show that a game $(N, v) \in \Gamma$ is totally balanced and overbalanced if and only if it is exactly balanced.

$(\Leftarrow)$ For $D \in \mathcal{N} \backslash\{N\}$, to show balancedness of $\left(D, v^{D}\right)$ it is sufficient to restrict attention to balanced vectors of weights where the weight on $D$ is equal to zero. This is obtained by setting the exactly balanced weights such that $\lambda_{N}=1, \lambda_{D}=-1$, and $\lambda_{C}=0$ for all $C \notin \mathcal{D}$. Balancedness of $(N, v)$ is obtained by considering exactly balanced vectors of weights where all the weights are non-negative. Using exactly balanced vectors of weights with $\lambda_{N}=0$ and $\lambda_{D} \leq 0$ implies overbalancedness of $(N, v)$.

$(\Rightarrow)$ Take any exactly balanced vector of weights $\left(\lambda_{C}\right)_{C \in \mathcal{N}}$ with $\lambda_{D} \in \mathbb{R}, \lambda_{C} \in \mathbb{R}_{+}$ for $C \in \mathcal{N} \backslash\{D\}$, and

$$
\sum_{C \in \mathcal{N}} \lambda_{C} a(C)=a(N) .
$$

It follows immediately that $\lambda_{N} \leq 1$. We discuss two cases depending on the value of $\lambda_{N}$.

1. $\lambda_{N}<1$. In this case (3) can be rearranged as

$$
\sum_{C \in \mathcal{N} \backslash\{N\}} \frac{\lambda_{C}}{1-\lambda_{N}} a(C)=a(N) .
$$

If $\lambda_{D} \leq 0$, then exact balancedness is implied by overbalancedness of $(N, v)$, otherwise by balancedness of $(N, v)$.

2. $\lambda_{N}=1$. If $\lambda_{D} \geq 0$, then exact balancedness is trivially satisfied since (3) implies $\left(\lambda_{C}\right)_{C \in \mathcal{N} \backslash\{N\}}=0$. If $\lambda_{D}<0$, then (3) can be rearranged as

$$
\sum_{C \in \mathcal{N} \backslash\{D, N\}} \frac{\lambda_{C}}{-\lambda_{D}} a(C)=a(D) .
$$

Note that (5) implies that $\lambda_{C}=0$ for all $C \notin \mathcal{D}$ and exact balancedness follows from balancedness of $\left(D, v^{D}\right)$.

Theorem 3.7 characterizes exact games as being the only totally balanced and overbalanced games. It follows immediately that an exact game is totally balanced, which was also shown by Schmeidler (1972). The result shows that exact games constitute the subset of totally balanced games that satisfies the extra condition of overbalancedness, where no weight is put on the grand coalition and one weight should be non-positive when checking the "normal" balancedness of the game.

Schmeidler (1972) characterizes exact games as follows (see Derks and Reijnierse (1998), Theorem 7). 
Theorem 3.8 (Schmeidler 1972) A game $(N, v) \in \Gamma$ is exact if and only if for every $D \in \mathcal{N} \backslash\{N\}$, for each vector $\left(\gamma_{C}\right)_{C \in \mathcal{N}}$ such that $\gamma_{C} \in \mathbb{R}_{+}$for all $C \in \mathcal{N}$ and $\sum_{C \in \mathcal{N} \backslash\{N\}} \gamma_{C} a(C)=a(D)+\gamma_{N} a(N)$ we have $\sum_{C \in \mathcal{N} \backslash\{N\}} \gamma_{C} v(C) \leq v(D)+$ $\gamma_{N} v(N)$.

Next, we provide a direct proof of the equivalence of Schmeidler's characterization of exactness and the characterization in Theorem 3.7.

First, we show that Schmeidler's characterization of exactness implies total balancedness and overbalancedness. That $\left(D, v^{D}\right)$ is balanced for $D \in \mathcal{N} \backslash\{N\}$ follows by setting $\gamma_{N}=0$ and $\gamma_{C}=0$ for all $C \notin \mathcal{D}$. Take a balanced vector of weights $\left(\lambda_{C}\right)_{C \in \mathcal{N}}$. Balancedness of $(N, v)$ follows from weights such that $\gamma_{N}=1$ and $\gamma_{D}=\lambda_{D}+1 \geq 1$. Overbalanced weights satisfy the equality

$$
\sum_{C \in \mathcal{N} \backslash\{D, N\}} \mu_{C} a(C)=a(N)+\mu_{D} a(D) .
$$

The case where $\mu_{D}=0$ follows from Schmeidler's characterization with $\gamma_{N}=1$ and $\gamma_{D}=1$. In case $\mu_{D}>0$, (6) is equivalent to

$$
\sum_{C \in \mathcal{N} \backslash\{D, N\}} \frac{\mu_{C}}{\mu_{D}} a(C)=\frac{1}{\mu_{D}} a(N)+a(D) .
$$

It is now easily seen that this case is implied by Schmeidler's characterization when we choose $\gamma_{D}=0, \gamma_{N}=1 / \mu_{D}$, and $\gamma_{C}=\mu_{C} / \mu_{D}$ for $C \in \mathcal{N} \backslash\{D, N\}$.

Second, we show that total balancedness and overbalancedness imply Schmeidler's characterization of exactness. The balancing weights of Schmeidler satisfy

$$
\sum_{C \in \mathcal{N} \backslash\{N\}} \gamma_{C} a(C)=a(D)+\gamma_{N} a(N) .
$$

In this case Schmeidler's condition is $\sum_{C \in \mathcal{N} \backslash\{N\}} \gamma_{C} v(C) \leq v(D)+\gamma_{N} v(N)$. If $\gamma_{N}=0$, then (7) implies that $\gamma_{C}=0$ for all $C \notin \mathcal{D}$ and thus the balancedness of $\left(D, v^{D}\right)$ gives rise to Schmeidler's condition. If $\gamma_{N}>0$ and $\gamma_{D} \geq 1$, then (7) is equivalent to

$$
\sum_{C \in \mathcal{N} \backslash\{D, N\}} \frac{\gamma_{C}}{\gamma_{N}} a(C)+\frac{\gamma_{D}-1}{\gamma_{N}} a(D)=a(N),
$$

and Schmeidler's condition follows from balancedness. If $\gamma_{N}>0$ and $\gamma_{D}<1$, then (7) is equivalent to

$$
\sum_{C \in \mathcal{N} \backslash\{D, N\}} \frac{\gamma_{C}}{\gamma_{N}} a(C)=a(N)+\frac{1-\gamma_{D}}{\gamma_{N}} a(D),
$$

so Schmeidler's condition follows from overbalancedness. 
For a coalition $C \in \mathcal{N}$ let $|C|$ denote the number of players involved in the coalition. Azrieli and Lehrer (2005) give the following necessary and sufficient conditions for exactness.

Theorem 3.9 (Azrieli and Lehrer, 2005, Proposition 2) A game $(N, v) \in \Gamma$ is exact if and only if for every $D \in \mathcal{N}$, for each vector $\left(\alpha_{C}\right)_{C \in \mathcal{N}}$ such that $\alpha_{C} \in \mathbb{R}_{+}$for all $C \in \mathcal{N}, \sum_{C \in \mathcal{N}} \alpha_{C}=1, \beta \in[0,1]$ and $\sum_{C \in \mathcal{N}} \alpha_{C} \frac{a(C)}{|C|}=\beta \frac{a(D)}{|D|}+(1-\beta) \frac{a(N)}{|N|}$ we have $\sum_{C \in \mathcal{N}} \alpha_{C} \frac{v(C)}{|C|} \leq \beta \frac{v(D)}{|D|}+(1-\beta) \frac{v(N)}{|N|}$.

Notice that in Theorem 3.9 if $D=N$, we have the usual balancedness condition expressed in terms of average worth. It is however not straightforward to give an interpretation to the condition in terms of average worth.

One can also show that the characterization by Azrieli and Lehrer (2005) is equivalent to the characterization by total balancedness and overbalancedness.

Our characterizations of exact games differ from the ones by Schmeidler (1972) and by Azrieli and Lehrer (2005) in that a weight of one is put on $a(N)$. This way we can employ the usual interpretation of balancedness, where players allocate one unit of time over the various coalitions, but now players in the coalition with a negative weight work overtime. In doing so, to satisfy our conditions working for the grand coalition and working overtime in the coalition with the negative weight should be the best choice players can make.

\section{Applications of exact balancedness}

In this section we show a number of applications of the condition of exact balancedness (Definition 3.3). First we demonstrate that exact games are convex for the grand coalition (Definition 2.5) and the class of totally exact games (Definition 2.6) coincides with the class of convex games (Definition 2.4). Then we discuss games with at most three players, where exact games turn out to be convex. Finally, we present an example of a game with four players that is totally balanced, convex for the grand coalition, but not exact.

Proposition 4.1 If the game $(N, v)$ is exact, then it is convex for the grand coalition, $\Gamma_{\mathrm{e}} \subseteq \Gamma_{\mathrm{cg}}$.

Proof Take two coalitions $S, T \in 2^{N}$ such that $S \cup T=N$. Let $D=S \cap T$. Since $(N, v)$ is exact, by Theorem 3.5 it is exactly balanced. We define an exactly balanced vector of weights by setting $\lambda_{S}=1, \lambda_{T}=1$, and $\lambda_{D}=-1$. All other balancing weights are set to zero. Notice that this constitutes an exactly balanced vector of weights, since $a(S)+a(T)-a(D)=a(N)$. By exact balancedness we have that $v(S)+v(T)-v(D) \leq v(N)$, that is $v(S)+v(T) \leq v(N)+v(S \cap T)$.

Theorem 4.2 A game is totally exact if and only if it is convex, $\Gamma_{\mathrm{te}}=\Gamma_{\mathrm{c}}$.

Proof $(\Rightarrow)$ Since by Proposition 4.1 exact games are convex for the grand coalition, totally exact games are convex for all coalitions, that is they are convex.

$(\Leftarrow)$ It is known that convex games are exact (Schmeidler 1972). Since a subgame of a convex game is also convex, we have total exactness. 
The following result claims that a game with at most three players that is totally balanced and convex for the grand coalition has to be convex.

Proposition 4.3 Assume that there are at most three players. If the game $(N, v)$ is totally balanced and convex for the grand coalition, then it is convex.

Proof For one or two player games the claim requires no proof. Consider the case with three players. For coalitions $S, T \in 2^{N}$ such that $S \cup T=N$ convexity follows from the convexity for the grand coalition. When $S \cap T=\{\varnothing\}, S \cup T=C$, where $C$ is a two-player coalition, convexity follows from total balancedness. When $S \subseteq T$, where $T$ is a two-player coalition, convexity follows since $S \cap T=S$ and $S \cup T=T$.

Exact games are totally balanced (Theorem 3.7) and convex for the grand coalition (Proposition 4.1): $\Gamma_{\mathrm{e}} \subseteq \Gamma_{\mathrm{tb}} \cap \Gamma_{\mathrm{cg}}$. Since a convex game is exact, using Proposition 4.3 we have the following corollary.

Corollary 4.4 Assume that there are at most three players. Then a game is exact if and only if it is convex.

There exist games that are convex for the grand coalition, but that are not totally balanced, and games that are totally balanced, but not convex for the grand coalition, so $\Gamma_{\mathrm{tb}} \cap \Gamma_{\mathrm{cg}} \subsetneq \Gamma_{\mathrm{tb}}$ and $\Gamma_{\mathrm{tb}} \cap \Gamma_{\mathrm{cg}} \subsetneq \Gamma_{\mathrm{cg}}$. Theorem 3.7 and Proposition 4.1 imply that $\Gamma_{\mathrm{e}} \subseteq \Gamma_{\mathrm{tb}} \cap \Gamma_{\mathrm{cg}}$. We consider next the question whether there are games that are totally balanced and convex for the grand coalition, but not exact. Using the intuition behind exact balancedness we provide an example of such a game in Example 4.5, therefore $\Gamma_{\mathrm{e}} \subsetneq \Gamma_{\mathrm{tb}} \cap \Gamma_{\mathrm{cg}}$.

Example 4.5 We present a game that is totally balanced and convex for the grand coalition, but not exact. By Proposition 4.3 we need at least four players. Consider the following game with four players. Let

$$
\begin{aligned}
v(\{1\}) & =v(\{2\})=v(\{3\})=v(\{4\})=0, \\
v(\{1,2\}) & =v(\{1,3\})=v(\{1,4\})=1, \\
v(\{2,3\}) & =v(\{2,4\})=v(\{3,4\})=0, \\
v(\{1,2,3\}) & =v(\{1,2,4\})=v(\{1,3,4\})=1, \\
v(\{2,3,4\}) & =0, \\
v(\{1,2,3,4\}) & =2 .
\end{aligned}
$$

The game $(N, v)$ is totally balanced, since every subgame $\left(C, v^{C}\right)$ where $C$ contains player 1 has a core element where player 1 receives $v(C)$ and the other players nothing, and the zero vector is a core element of all other subgames. The game $(N, v)$ is convex for the grand coalition as $v(N)=2$ weakly exceeds the sum of the value of any other two coalitions. We show that the game $(N, v)$ is not exactly balanced. Let $D=(\{1\})$ and consider the weights $\lambda_{\{1,2\}}=\lambda_{\{1,3\}}=\lambda_{\{1,4\}}=1, \lambda_{\{1\}}=-2$, and set all other weights equal to zero. These weights constitute an exactly balanced vector of weights, since $a(\{1,2\})+a(\{1,3\})+a(\{1,4\})-2 a(\{1\})=a(\{1,2,3,4\})$. However, $v(\{1,2\})+v(\{1,3\})+v(\{1,4\})-2 v(\{1\})=3>2=v(\{1,2,3,4\})$, thus $v$ is not exact. 


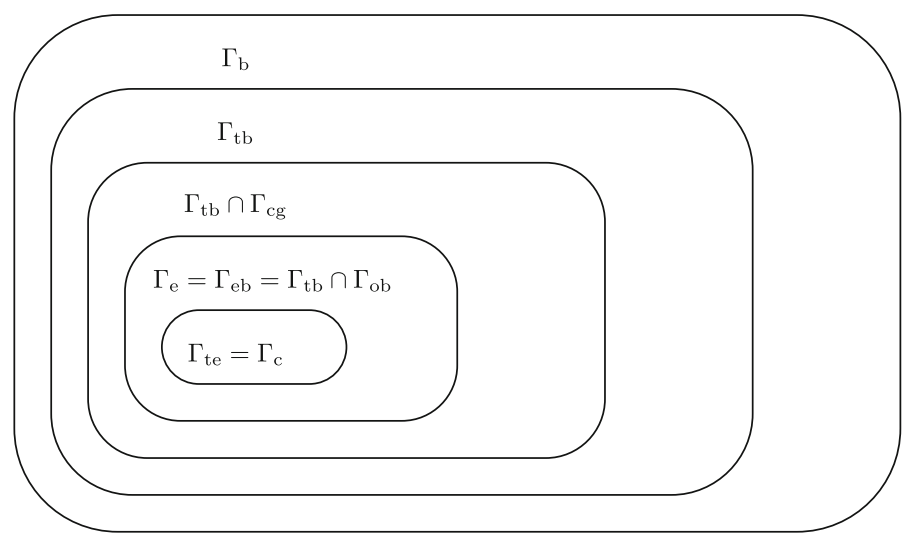

Fig. 1 Subsets of balanced games

The relationships between the various classes of games are summarized in Fig. 1 . As a future line of research, one can look for generalizations of exact balancedness for non-transferable utility (NTU) games. The first step was done by Csóka et al. (2011) by defining exactness and relating it to the various notions of convexity in the NTU setting.

Acknowledgments Péter Csóka thanks funding by the project TÁMOP-4.2.1/B-09/1/KMR-2010-0005 and the Hungarian Academy of Sciences under its Momentum programme (LD-004/2010). P. Jean-Jacques Herings would like to thank the Netherlands Organisation for Scientific Research (NWO) for financial support. László Á. Kóczy thanks funding by the European Commission under the Marie Curie Intra-European Fellowship MEIF-CT-2004-011537, the Reintegration Grant PERG-GA-2008-230879 as well as by the OTKA (Hungarian Scientific Research Fund) for the project NF-72610 and the support of the Hungarian Academy of Sciences under its Momentum programme (LD-004/2010).

Open Access This article is distributed under the terms of the Creative Commons Attribution Noncommercial License which permits any noncommercial use, distribution, and reproduction in any medium, provided the original author(s) and source are credited.

\section{References}

Azrieli Y, Lehrer E (2005) Concavification and convex games. Working Paper

Biswas AK, Parthasarathy T, Potters JAM, Voorneveld M (1999) Large cores and exactness. Games Econ Behav 28:1-12

Bondareva ON (1963) Some applications of linear programming methods to the theory of cooperative games (in Russian). Problemy Kybernetiki 10:119-139

Branzei R, Tijs S, Zarzuelo J (2009) Convex multi-choice games: characterizations and monotonic allocation schemes. Eur J Oper Res 198:571-575

Calleja P, Borm P, Hendrickx R (2005) Multi-issue allocation situations. Eur J Oper Res 164:730-747

Carpente L, Casas-Méndez B, García-Jurado I, van den Nouweland A (2010) The truncated core for games with upper bounds. Int J Game Theory 39:645-656

Casas-Méndez B, García-Jurado I, van den Nouweland A, Vázquez-Brage M (2003) An extension of the $\tau$-value to games with coalition structures. Eur J Oper Res 148:494-513

Csóka P, Herings PJJ, Kóczy LÁ (2009) Stable allocations of risk. Games Econ Behav 67(1):266-276

Csóka P, Herings PJJ, Kóczy LÁ, Pintér M (2011) Convex and exact games with non-transferable utility. Eur J Oper Res 209:57-62 
Derks J, Reijnierse H (1998) On the core of a collection of coalitions. Int J Game Theory 27:451-459

Dubey P, Shapley LS (1984) Totally balanced games arising from controlled programming problems. Mathematical Program 29:245-267

Fang Q, Fleischer R, Li J, Sun X (2010) Algorithms for core stability, core largeness, exactness, and extendability of flow games. Front Math Chin 5:47-63

Kalai E, Zemel E (1982a) Generalized network problems yielding totally balanced games. Oper Res 30: 998-1008

Kalai E, Zemel E (1982b) Totally balanced games and games of flow. Math Oper Res 7:476-478

Legut J (1990) On totally balanced games arising from cooperation in fair division. Games Econ Behav 2:47-60

Owen G (1975) On the core of linear production games. Math Program 9:358-370

Predtetchinski A, Herings PJJ (2004) A necessary and sufficient condition for the non-emptiness of the core of a non-transferable utility game. J Econ Theory 116:84-92

Schmeidler D (1972) Cores of exact games. J Math Anal Appl 40:214-225

Shapley LS (1967) On balanced sets and cores. Nav Res Logist Q 14:453-460

Shapley LS (1971) Cores of convex games. Int J Game Theory 1:11-26

Shapley LS, Shubik M (1969) On market games. J Econ Theory 1:9-25

Tijs S, Parthasarathy T, Potters J, Prassad VR (1984) Permutation games: another class of totally balanced games. OR Spektrum 6:119-123

van Velzen B, Hamers H, Solymosi T (2008) Core stability in chain-component additive games. Games Econ Behav 62:116-139 